\title{
CURSO DE POST-GRADUADOS
}

\author{
SOBRE EL METODO PSICOPROFILACTICO
}

Como oportunamente fue avisado, a principios del mes de agosto del presente, se efectuó el primer curso de post-graduados sobre los métodos psicofísicos de analgesia obstétrica, orga. nizado por el Equipo Médico de Estudios Psicoprofilácticos de Analgesia Obstétrica.

Este curso, dedicado especialmente a médicos de fuera de la capital, se desarrolló con cupo completo, como podrá apreciarse por la siguiente lista de los distinguidos colegas asistentes: doctor Alberto Espejo, de Duitama; doctor Gustavo Romero Hernández, de Tibaná; doctores Fernando Valencia, Humberto Góngora, Germán Muñoz y Fabio Mejía, de Manizales; doctores Fernando Navas Uribe, Hugo Flórez y Fabián Jiménez, de Barranquilla; doctores Ignacio Londoño, Carlos Arboleda, Alfredo Londoño y Hugo García de Pereira; doctor Libardo Arango, de Medellín; doctor Germán Motta, de Bucaramanga; doctor Mario Bueno Barrios, de Ibagué; doctor Antonio M. Martínez, de Cartagena; doctor Luis Nova, del Socorro; doctor Luis Carlos González, de Villavicencio; doctor Enrique Vargas, de Paz de Río; señorita María del C. Pinzón, de la Escuela Nacional de Enfermería; señorita Ruby Palacios, de la Escuela Superior de Higiene; doctores Rodulfo Camero, Nélson Bruno, Jaime Calle P., Carlos Ramírez, Hernán Rodríguez A. y Juan Mendoza Vega, de Bogotá.

Las actividades del curso tuvieron lugar casi en su totalidad en la Clínica Palermo. Va para un año que el Equipo Médico de Estudios Psicoprofilácticos de Analgesia Obstétrica, inició en esta Clínica la aplicación del método en pacientes particulares, contando a su favor no sólo con la dotación magnífica y excepcio_ nal de la Clínica, sino con el apoyo y la amplia colaboración de la Reverenda Madre Superiora, del Director de la Clínica doctor José M. Huertas y de las Hermanas y médicos internos de la Sección de Maternidad. 
Puede decirse que este curso ha sido el resultado de la experiencia adquirida no solamente en un año de trabajo y estudio en pacientes privados de la Clínica Palermo, sino de la práctica particular de los Obstetras integrantes del Equipo antes de su organización en grupo.

El siguiente fue el programa del curso:

Agosto 19

Conf. I. Doctor Guillermo López E.-Introducción al estudio del método. 9 a. $\mathrm{m}$.

Conf. II. Doctor Belisario Calderón. Teoría de Pavlov. 10 a. m. Conf. III. Doctor Miguel A. Fernández Bastidas. Teoría de Read. 11 a. m.

Conf. IV. Doctor Juan B. Castaño C. Aspectos emocionales del embarazo. 3 p. $m$.

Conf. V. Doctor Alvaro Villar. Nociones de Psicoterapia en relación con el estado grávido. 4. p. $\mathrm{m}$.

Conf. VI. Doctor Miguel A. Fernández Bastidas. Cuidados prenatales en relación con el método Psicoprofiláctico. $5 \mathrm{p} . \mathrm{m}$.

9 p. m. Película. Mesa Redonda (Consultorio Especialistas).

\section{Agosto 2:}

Conf. VII. Doctor G. López Escobar. Relajación y métodos afines. 9. a. $\mathrm{m}$.

Conf. VIII. Doctor Carlos R. Silva M. Kinesiología y Obstetri_ cia. 10 a. m.

Conf. IX. Señora Cecilia de Mojica. Programa de ejercicios. Película. 11 a. $\mathrm{m}$.

Conf. X. Doctor Alberto Gómez T. Programa de clases teóricas. 3 p. $\mathrm{m}$.

Conf. XI. Doctor Hernando Amaya L. Condiciones para el buen resultado del método Psicoprofiláctico. $4 \mathrm{p} . \mathrm{m}$.

Conf. XII. Doctor Carlos R. Silva M. Conducta en el parto. 6 p. m.

\section{Agosto 3:}

Conf. XIII. Doctor Guillermo Lesmes A. Papel del pediatra en el método Psicoprofiláctico. 8 a. $\mathrm{m}$.

Conf. XIV. Doctor G. López Escobar. Ventajas del método para la madre. 9 a. $\mathrm{m}$.

Conf. XV. Doctor Belisario Calderón. Clasificación de resultados. 10 a. m. 
Conf. XVI. Doctor Carlos R. Silva M. Plan de difusión del método Psicoprofiláctico. 11 a. $\mathrm{m}$.

$12 \mathrm{~m}$. Mesa Redonda (Clínica Palermo).

1 p. m. Clausura del Curso. Almuerzo.

Los médicos asistentes recibieron programas suficientemente ilustrados sobre desarrollo de clases teóricas y sobre práctica de Kinesiterapia obstétrica. Las conferencias fueron bastante objetivas, lo que unido a la presentación de dos películas muy demostrativas, contribuyó a que los cursillistas captaran el método fácilmente. Se espera que en esta forma no encuentren mavores tropiezos para la aplicación del método en sus respectivos medios.

Una de las finalidades del Equipo consiste en la difusión del método entre médicos, enfermeras y estudiantes de medicina. En la conferencia que el curso dedicó a este punto se hizo resal_ tar la necesidad de una difusión seria y científica ante todo para evitar el fracaso del método debido a charlatanería o a su práctica por personas no suficientemente ilustradas, o por obstetras aficionados.

Fueron proyectadas dos películas: una sobre Kinesioterapia y otra sobre "El parto indoloro por el método Psicoprofiláctico". Las dos películas fueron filmadas por personal del equipo, una en la Clínica Palermo y la otra en el Hospital de San José, con la colaboración del Equipo de Psicoprofilaxia de dicho Hospital. Igualmente las pacientes que figuran en las películas fueron preparadas por los mencionados Equipos. Como varios colegas se han manifestado interesados en las películas, se les informa sobre sus características: están en blanco y negro, en $8 \mathrm{~mm}$. El Equipo escogió este tipo de película por la facilidad y econom'a que ella representa para ser proyectada sin ninguna complicación en cualquier medio. Para mayores detalles, dirigirse a la Secretaría del Equipo (Calle 63-A, número 10-48, D. E.).

Puede decirse que los resultados del curso fueron satisfactorios a juzgar por los informes personales escritos, presentados por cada uno de los cursillistas. El Equipo se propone organizar un segundo curso teórico-práctico, más extenso que éste en cola. boración con el Equipo de Psicoprofilaxia del Hospital San José, para principios del año próximo. Sobre este particular se dará información oportuna. 